\title{
An amine protecting group deprotectable under nearly neutral oxidative conditions
}

\author{
Shahien Shahsavari ${ }^{1}$, Chase McNamara ${ }^{1}$, Mark Sylvester ${ }^{1}$, Emily Bromley ${ }^{1}$, \\ Savannah Joslin ${ }^{1}$, Bao-Yuan Lu ${ }^{2}$ and Shiyue Fang ${ }^{* 1}$
}

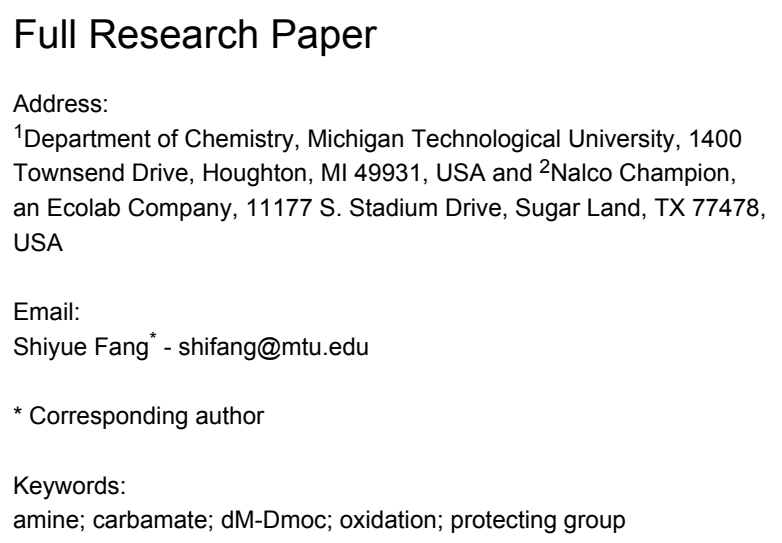

amine; carbamate; dM-Dmoc; oxidation; protecting group

Beilstein J. Org. Chem. 2018, 14, 1750-1757. doi:10.3762/bjoc. 14.149

Received: 16 March 2018

Accepted: 03 July 2018

Published: 13 July 2018

Associate Editor: B. Stoltz

(C) 2018 Shahsavari et al.; licensee Beilstein-Institut. License and terms: see end of document.

\begin{abstract}
The 1,3-dithiane-based dM-Dmoc group was studied for the protection of amino groups. Protection was achieved under mild conditions for aliphatic amines, and under highly reactive conditions for the less reactive arylamines. Moderate to excellent yields were obtained. Deprotection was performed by oxidation followed by treating with a weak base. The yields were good to excellent. The new amino protecting group offers a different dimension of orthogonality in reference to the commonly used amino protecting groups in terms of deprotection conditions. It is expected to allow a collection of transformations to be carried out on the protected substrates that are unattainable using any known protecting groups.
\end{abstract}

\section{Introduction}

In multistep organic synthesis, amino groups usually have to be protected [1]. Protecting groups for the purpose mainly include those deprotectable by acid (e.g., tert-butyloxycarbonyl (Boc) group) [2-4], base (e.g., 9-fluorenylmethyloxycarbonyl (Fmoc) group and trifluoroacetyl group) [5-7], catalytic hydrogenation (e.g., benzyl group) [8], photoirradiation (e.g., 2-nitrophenylethyl carbamate and 6-nitroveratryl carbamate) $[9,10]$ and fluoride (e.g., trimethylsilylethyloxycarbonyl (Teoc) group) [11,12]. The 1,3-dithian-2-ylmethoxycarbonyl (Dmoc) group first reported by Kunz and co-workers provides a different dimension of orthogonality of amine protection in terms of deprotection conditions [13-17]. This group was deprotected under oxidative conditions under which the commonly used Boc, Fmoc, benzyl and Teoc groups could potentially survive. Oxidation was achieved by hydrogen peroxide in the presence of an ammonium molybdate catalyst. Recently, we reported the use of the Dmoc group for amine protection in automated solidphase oligodeoxynucleotide (ODN) synthesis [18]. For deprotection, we found that sodium periodate could effectively oxidize multiple Dmoc functions in the ODNs to achieve com- 
plete deprotection. Under these oxidative conditions, oxidation of the ODN was not observed. The mild deprotection conditions allowed us to introduce sensitive functionalities into ODNs, which are otherwise impossible or highly difficult to achieve [18]. In addition, we also investigated the potential of the dimethyl-1,3-dithian-2-ylmethyl (dM-Dim) group for orthogonal carboxylic acid protection [19]. To further explore the use of the 1,3-dithiane function as protecting group in organic synthesis, here we report the results of our studies on the use of the dimethyl-1,3-dithian-2-ylmethoxycarbonyl (dM-Dmoc) group for amine protection (Scheme 1). Compared with the Dmoc group, the dM-Dmoc group is expected to be more stable under nucleophilic conditions, which will allow many transformations including base hydrolysis of esters and amides, hydride reduction of carbonyl compounds, and a wide range of nucleophilic substitution reactions to be carried out without losing the protection. With Dmoc protection, such transformations would be unattainable or require fine tuning of reaction conditions to keep the protection. In addition, the side product $\mathbf{2}$ from deprotection of dM-Dmoc is less likely to act as a Michael acceptor to react with the amine product than $\mathbf{1}$ from deprotection of Dmoc due to its higher steric hindrance. Such side reactions could be a serious issue in some situations [18].

\section{Results and Discussion}

To protect amines, compound $\mathbf{4}$ was prepared readily by reacting deprotonated 1,3-dithiane with acetone followed by treating with $p$-nitrophenylchloroformate (see experimental section). The compound is stable, which allows easy purification and storage. However, we expected that it could react with amines under suitable conditions. Using benzylamine (3a) as the model substrate, we tested a variety of reaction conditions to form the dM-Dmoc protected carbamate 5a (see Table 1 for structures). These include using different solvents such as THF, DCM, acetonitrile and toluene, and different bases such as DIPEA, pyridine and trimethylamine. We found that the condi- tions most suitable for the reaction were to react one equivalent amine with one equivalent of $\mathbf{4}$ in the solvent THF using five equivalents of DIPEA as the base. At room temperature, the reaction could complete within eight hours.

After suitable conditions for protection of amines with dM-Dmoc were identified, we investigated the substrate scope of the reaction. As shown in Table 1, primary aliphatic amines including 3a-d gave good to excellent isolated yields of carbamates 5a-d (Table 1, entries 1-4). Under these conditions, however, secondary aliphatic amines could not react or could react but gave very low yields. We tried a variety of other conditions such as deprotonating the amine followed by reacting with 4 and heating excess amine with $\mathbf{4}$ without any solvent but failed to identify one that could afford useful yields. We also tried to use the optimized conditions for the protection of aliphatic primary amines to protect arylamines, but found that arylamines were not reactive enough for the reaction. Therefore, for protecting arylamines, we used conditions for the formation of hindered $O$-tert-alkyl $N$-arylcarbamates we reported earlier [20]. Treating one equivalent $3 \mathbf{e}$ with two equivalents LDA and one equivalent 4 in THF gave the desired arylamine dM-Dmoc carbamate 5e in synthetically useful yield (Table 1, entry 5). Three additional arylamines were also tested, which include the two heterocyclic arylamines $\mathbf{3} \mathbf{g}$ and $\mathbf{3 h}$, all gave synthetically useful yields of the aryl carbamate products $\mathbf{5 f}-\mathbf{h}$ (Table 1, entries 6-8). Finally, to investigate the suitability of the dM-Dmoc group for protecting amino acids, phenylalanine (3i) was selected to react with $\mathbf{4}$ to give $\mathbf{5 i}$ (Table 1, entry 9). The general conditions for aliphatic amine protection were used, but due to the low solubility of $\mathbf{3 i}$ in THF, DMSO was used as the solvent. Compound $\mathbf{5 i}$ was obtained in $80 \%$ isolated yield.

For deprotection of dM-Dmoc protected amines, we used the conditions we developed earlier for the deprotection of Dmoc protected ODNs directly without making additional efforts to

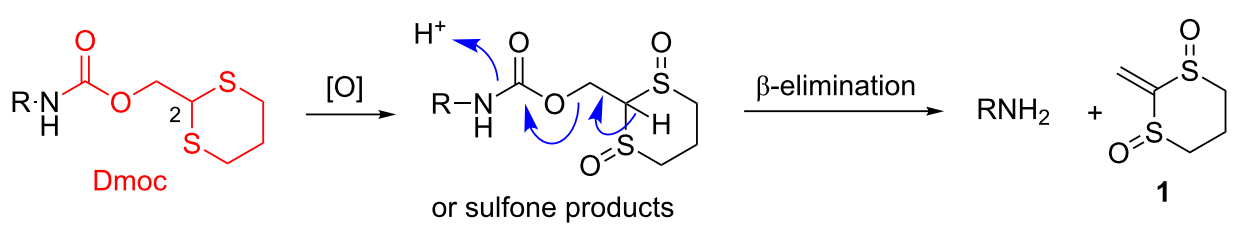

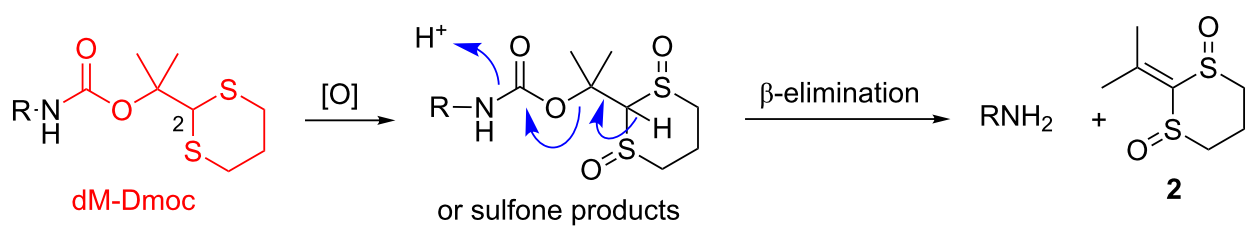


Table 1: Protection of amines with dM-Dmoc and deprotection. ${ }^{\text {a }}$

3

1

$$
\mathrm{Ph} \mathrm{NH}_{2}
$$

3a

$n-\mathrm{BuNH}_{2}$

$3 \mathrm{~b}$

3<smiles>C=CCN</smiles><smiles>[13CH3][Si](OCCN)(c1ccccc1)c1ccccc1</smiles>

$\mathrm{PhNH}_{2}$

3e
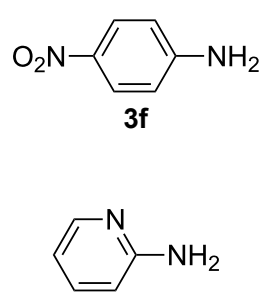

$3 g$

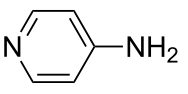

$3 h$<smiles>N[C@@H](Cc1ccccc1)C(=O)O</smiles><smiles>CC(C)(OC(=O)NCc1ccccc1)C1SCCCS1</smiles>

3a (76\%)

5a (92\%)<smiles>[3H][10B]NC(=O)OC(C)(C)C1SCCCS1</smiles>

5b $(89 \%)$<smiles>C=CCNC(=O)OC(C)(C)C1SCCCS1</smiles>

3c $(55 \%)$

5c $(72 \%)$<smiles>[3H][Si](OCCNC(=O)OC(C)(C)C1SCCCS1)(c1ccccc1)c1ccccc1</smiles>

3d (88\%)

5d $(97 \%)$<smiles>CC(C)(OC(=O)Nc1ccccc1)C1SCCCS1</smiles>

5e $(46 \%)$<smiles>CC(C)(OC(=O)Nc1ccc([N+](=O)[O-])cc1)C1SCCCS1</smiles><smiles>CC(C)(OC(=O)Nc1ccccn1)C1SCCCS1</smiles>

5g (57\%)<smiles>CC(C)(OC(=O)Nc1ccncc1)C1SCCCS1</smiles>

5h $(52 \%)$<smiles>CC(C)(OC(=O)N[C@@H](Cc1ccccc1)C(=O)O)C1SCCCS1</smiles>

$5 \mathbf{i}(80 \%)$

aReaction conditions: For converting $\mathbf{3 a} \mathbf{a}-\mathbf{d}$ to $\mathbf{5 a} \mathbf{a}-\mathbf{d}, \mathbf{3}$ (1 equiv), $\mathbf{4}$ (1 equiv), DIPEA (5 equiv), THF, rt, 8 h. For $\mathbf{3 e - h}$ to $\mathbf{5 e - h}, \mathbf{3}$ ( 1 equiv), $\mathbf{4}$ ( 1 equiv), LDA (2 equiv), THF, $-78{ }^{\circ} \mathrm{C}$ to rt, $8 \mathrm{~h}$. For $\mathbf{3 i}$ to $\mathbf{5 i}, \mathbf{3 i}$ (1 equiv), 4 (1 equiv), DIPEA (5 equiv), DMSO, rt, 8 h. For 5 to 3,5 ( 1 equiv), NalO 4 (10 equiv), $\mathrm{THF} / \mathrm{H}_{2} \mathrm{O}(\mathrm{v} / \mathrm{v} 1: 1)$, rt, $12 \mathrm{~h}$; then $\mathrm{K}_{2} \mathrm{CO}_{3}$ (10 equiv), $\mathrm{MeOH}\left(\mathrm{MeOH} / \mathrm{H}_{2} \mathrm{O}\right.$ for $\mathbf{5 i}$ to $\left.\mathbf{3 i}\right)$, rt, $1 \mathrm{~h}$. Isolated yields were obtained in all cases except for $\mathbf{3 i}$, for which the yield was determined with RP-HPLC. 
evaluate other conditions [18]. These conditions could be superior to reported conditions $[13,15,17,21]$ because they do not require transition metal catalysts or any special devices such as an electrochemical cell. Therefore, the dM-Dmoc carbamates were first oxidized with sodium periodate at room temperature. After removing the excess oxidizing agent and other inorganic salts by filtration, $\beta$-elimination to give the amine products was initiated with the weak base potassium carbonate at room temperature. The products were then purified with flash column chromatography. As shown in Table 1, the yields of the deprotection ranged from $48 \%$ to $88 \%$. Among them, $3 \mathbf{a}$ and $\mathbf{3 d}$, which are aliphatic amines, gave better yields (Table 1, entries 1 and 4). Compounds $\mathbf{3 b}$ and $\mathbf{3 c}$ are also aliphatic amines, but their yields were lower. This might be caused by evaporation due to their low boiling points during work-up and purification. The arylamines were obtained in lower yields (Table 1, entries 5-8) compared with the aliphatic ones. Among the four arylamine examples, $\mathbf{5} \mathbf{g}$ and $\mathbf{5} \mathbf{h}$ contained a pyridine ring, which could be sensitive to oxidative conditions. However, it looked like that sodium periodate was benign to pyridine and some other nitrogen containing aromatic heterocycles [18]. The dM-Dmoc protected phenylalanine (5i) was deprotected under slightly different conditions (Table 1 , entry 9 ). In the $\beta$-elimination step, when methanol was used as the solvent as in the general deprotection procedure, no reaction occurred even after stirring overnight. This might be caused by the more favoured deprotonation of the carboxylic acid group by potassium carbonate, which made the starting material insoluble and prevented deprotonation of $\mathrm{H}-2$ in the oxidized 1,3-dithiane function. The problem was solved by using a solvent mixture of methanol and water. It is important to note that carrying out the deprotection reaction in one pot by performing the oxidation under basic conditions is not feasible. In theory, using the one pot approach, once the sulfides in dM-Dmoc were oxidized, $\beta$-elimination would follow to give the desired amine products directly. We tested the idea, and as expected, complex mixtures were formed. Reasons for the observation include oxidation of amine products by sodium periodate and its reduced products. In addition, we also found that oxidation of sulfides by sodium periodate was significantly slower under basic conditions than under neutral and acidic conditions.

To demonstrate the feasibility of selective deprotection of $\mathrm{dM}$-Dmoc protected amines in the presence of Boc protected ones, compound 6 [22] was reacted with 4 under the general aliphatic amine protection conditions to give the Boc and dM-Dmoc protected diamine 7 (Scheme 2). Selective removal of dM-Dmoc was simply achieved under the general deprotection conditions without any fine tuning of conditions. The desired Boc protected 6 was obtained in $80 \%$ isolated yield. To demonstrate the orthogonality of dM-Dmoc and Fmoc protections, compound 9 was prepared (Scheme 2). Compound 4 was

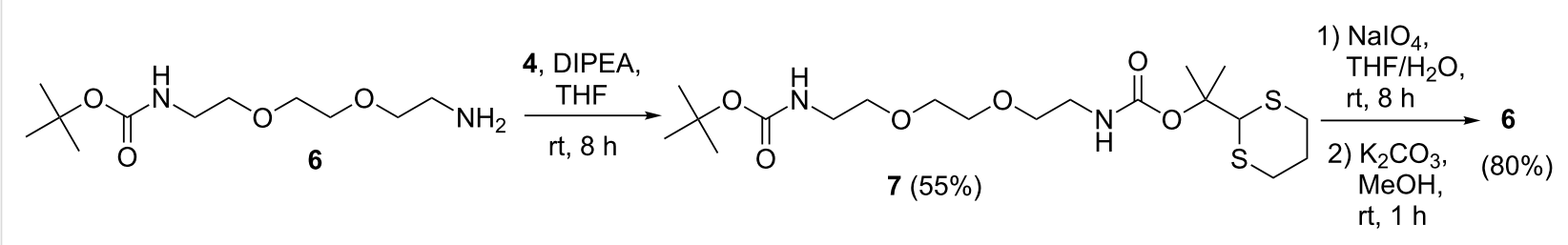<smiles>CC(C)(OC(=O)NCCOCCOCCN)C1SCCCS1</smiles><smiles>CC(C)(OC(=O)NCCOCCOCCNC(=O)OCC1c2ccccc2-c2ccccc21)C1SCCCS1</smiles>

$$
9 \stackrel{\begin{array}{l}
\text { 1) } \mathrm{NalO}_{4}, \mathrm{THF} / \mathrm{H}_{2} \mathrm{O}, \\
\mathrm{rt}, 8 \mathrm{~h}
\end{array}}{\stackrel{\text { 2) } \mathrm{K}_{2} \mathrm{CO}_{3}, \mathrm{MeOH},}{\mathrm{rt}, 1 \mathrm{~h}}}
$$<smiles>NCCOCCOCCNC(=O)OCC1c2ccccc2-c2ccccc21</smiles> 
reacted with 1,2-bis(2-aminoethoxy)ethane to give $\mathbf{8}$, which was reacted with $\mathrm{Fmoc}-\mathrm{Cl}$ to give the $\mathrm{dM}-\mathrm{Dmoc}$ and Fmoc protected diamine 9. We found that selective removal of Fmoc from 9 to give 8 could be achieved under typical Fmoc deprotection conditions involving piperidine. Selective removal of $\mathrm{dM}$-Dmoc was also simple; treating 9 under the standard dM-Dmoc deprotection conditions gave the Fmoc protected diamine $\mathbf{1 0}$ in $75 \%$ isolated yield (Scheme 2). The basic conditions involving potassium carbonate used to induce $\beta$-elimination of oxidized dM-Dmoc did not cause any loss of Fmoc protection.

\section{Conclusion}

In summary, we have demonstrated that dM-Dmoc could serve as a new protecting group for aliphatic and arylamines. This group could be removed under nearly neutral oxidative conditions, which are orthogonal to the commonly used conditions for deprotection of protected amines including acid, base, and catalytic hydrogenation. Compared to Dmoc, dM-Dmoc has the advantage of being stable under a wide range of basic and nucleophilic conditions. We expect that the new protecting group will find wide applications in multistep organic synthesis.

\section{Experimental}

General: All reactions were performed in oven-dried glassware under an argon atmosphere using standard Schlenk techniques. Reagents and solvents available from commercial sources were used as received unless otherwise noted. THF and $\mathrm{CH}_{2} \mathrm{Cl}_{2}$ were dried using an Innovative Technology Pure-Solv ${ }^{\mathrm{TM}}$ system. Acetone, pyridine, and diisopropylamine were distilled over $\mathrm{CaH}_{2}$ under nitrogen. Thin-layer chromatography (TLC) was performed using Sigma-Aldrich TLC plates, silica gel 60F-254 over glass support, $0.25 \mu \mathrm{m}$ thickness. Flash column chromatography was performed using SiliCycle silica gel, particle size $40-63 \mu \mathrm{m} .{ }^{1} \mathrm{H}$ and ${ }^{13} \mathrm{C}$ spectra were measured on Varian UNITY INOVA spectrometer at 400 and $100 \mathrm{MHz}$, respectively; chemical shifts $(\delta)$ were reported in reference to solvent peaks (residue $\mathrm{CHCl}_{3}$ at $\delta 7.24 \mathrm{ppm}$ for ${ }^{1} \mathrm{H}$ and $\mathrm{CDCl}_{3}$ at $\delta 77.00 \mathrm{ppm}$ for ${ }^{13} \mathrm{C}$ ).

2-(1,3-Dithian-2-yl)propan-2-ol: To a solution of 1,3-dithiane $(5.0 \mathrm{~g}, 41.6 \mathrm{mmol})$ in dry THF $(100 \mathrm{~mL})$ was slowly added $n$-BuLi (2.5 M in pentane, $15.7 \mathrm{~mL}, 41.6 \mathrm{mmol})$ at $-78{ }^{\circ} \mathrm{C}$ under argon. After stirring for $30 \mathrm{~min}$, freshly distilled acetone (3.0 $\mathrm{mL}, 41.6 \mathrm{mmol}$ ) was added dropwise, and stirring was continued for $1 \mathrm{~h}$. The reaction was quenched with sat. $\mathrm{NH}_{4} \mathrm{Cl}$ $(75 \mathrm{~mL})$ and extracted with EtOAc $(50 \mathrm{~mL} \times 2)$. The extracts were dried over anhydrous $\mathrm{Na}_{2} \mathrm{SO}_{4}$, filtered, and concentrated. The residue was purified by flash column chromatography $\left(\mathrm{SiO}_{2}, 4: 1\right.$ hexanes/EtOAc) to afford the title compound as a white amorphous solid $(6.24 \mathrm{~g}, 84 \%)$ : TLC $R_{\mathrm{f}}=0.3(4: 1$
hexanes/EtOAc); IR (thin film) v 3339, 2930, $1420 \mathrm{~cm}^{-1}$; ${ }^{1} \mathrm{H}$ NMR (400 MHz, $\left.\mathrm{CDCl}_{3}\right) \delta 1.32(\mathrm{~s}, 6 \mathrm{H}), 1.73-1.85(\mathrm{~m}, 1 \mathrm{H})$, 2.00-2.07 (m, 1H), $2.41(\mathrm{~s}, 1 \mathrm{H}), 2.78-2.90(\mathrm{~m}, 4 \mathrm{H}), 4.10(\mathrm{~s}$, 1H) $\mathrm{ppm} ;{ }^{13} \mathrm{C} \mathrm{NMR}\left(100 \mathrm{MHz}, \mathrm{CDCl}_{3}\right) \delta 25.9,27.4,30.9$, 61.0, $73.4 \mathrm{ppm}$; HRMS (ESI) $\mathrm{m} / \mathrm{z}:[\mathrm{M}+\mathrm{K}]^{+}$calcd for $\mathrm{C}_{7} \mathrm{H}_{14} \mathrm{OS}_{2} \mathrm{~K}, 217.0123$; found, 217.0121 .

\section{2-(1,3-Dithian-2-yl)propan-2-yl (4-nitrophenyl) carbonate}

(4): To a solution of 2-(1,3-dithian-2-yl)propan-2-ol (6.4 g, $36 \mathrm{mmol}, 1$ equiv) and pyridine (2.9 mL, $54 \mathrm{mmol}, 1.5$ equiv) in DCM $(100 \mathrm{~mL})$ was added $p$-nitrophenylchloroformate (7.2 g, $36 \mathrm{mmol}, 1$ equiv) at rt under argon. After stirring for $8 \mathrm{~h}$, the contents were poured into a separatory funnel and partitioned between EtOAc $(40 \mathrm{~mL})$ and $\mathrm{H}_{2} \mathrm{O}(80 \mathrm{~mL})$. The aqueous layer was extracted with DCM $(50 \mathrm{~mL} \times 2)$. The combined organic layer was dried over anhydrous $\mathrm{Na}_{2} \mathrm{SO}_{4}$, filtered and concentrated. Flash column chromatography $\left(\mathrm{SiO}_{2}, 9: 1\right.$ hexanes/EtOAc) gave 4 as a white amorphous solid (10.0 g, $81 \%)$ : TLC $R_{\mathrm{f}}=0.4(5: 1$ hexanes/EtOAc); IR (thin film) $v$ 3083, 2981, 1713, 1592, $1522 \mathrm{~cm}^{-1} ;{ }^{1} \mathrm{H}$ NMR $(400 \mathrm{MHz}$, $\left.\mathrm{CDCl}_{3}\right) \delta 1.70(\mathrm{~s}, 6 \mathrm{H}), 1.81-1.91(\mathrm{~m}, 1 \mathrm{H}), 2.11-2.18(\mathrm{~m}, 1 \mathrm{H})$, 2.92-2.95 (m, 4H), $4.98(\mathrm{~s}, 1 \mathrm{H}), 7.38(\mathrm{~d}, J=9.2 \mathrm{~Hz}, 2 \mathrm{H}), 8.26$ $(\mathrm{d}, J=6.9 \mathrm{~Hz}, 2 \mathrm{H}) \mathrm{ppm} ;{ }^{13} \mathrm{C} \mathrm{NMR}\left(100 \mathrm{MHz}, \mathrm{CDCl}_{3}\right) \delta 24.1$, 25.7, 30.8, 56.2, 86.9, 121.9, 125.1, 145.2, 150.0, 155.5 ppm; HRMS (ESI) $m / z$ : $[\mathrm{M}+\mathrm{K}]^{+}$calcd for $\mathrm{C}_{14} \mathrm{H}_{18} \mathrm{O}_{2} \mathrm{~S}{ }_{2} \mathrm{~K}, 321.0385$; found, 321.0404 .

\section{General procedure for dM-Dmoc protection of aliphatic} amines - synthesis of carbamates 5a-d: To a solution of an amine (0.292 mmol, 1 equiv) and DIPEA $(0.255 \mathrm{~mL}$, $1.46 \mathrm{mmol}, 5$ equiv) in dry THF $(10 \mathrm{~mL})$ was added $4(0.100 \mathrm{~g}$, $0.292 \mathrm{mmol}, 1$ equiv) at $\mathrm{rt}$ under argon. After stirring for $8 \mathrm{~h}$, the reaction was quenched with sat. $\mathrm{NH}_{4} \mathrm{Cl}(15 \mathrm{~mL})$. Organics were extracted with EtOAc $(10 \mathrm{~mL} \times 2)$. The extracts were dried over anhydrous $\mathrm{Na}_{2} \mathrm{SO}_{4}$, filtered, and concentrated. The carbamates 5a (column eluted with 3:1 hexanes/EtOAc; TLC $R_{\mathrm{f}}=0.5$, developed with $1: 1$ hexanes/EtOAc), $5 \mathbf{b}$ (6:1 hexanes/ EtOAc; $R_{\mathrm{f}}=0.5,3: 1$ hexanes/EtOAc), 5c $(6: 1$ hexanes/EtOAc; $R_{\mathrm{f}}=0.5,3: 1$ hexanes/EtOAc), and 5d (9:1 hexanes/EtOAc; $R_{\mathrm{f}}=0.5,6: 1$ hexanes/EtOAc) were purified with flash column chromatography $\left(\mathrm{SiO}_{2}\right)$.

\section{2-(1,3-Dithian-2-yl)propan-2-yl benzylcarbamate (5a):} Colorless oil (0.084 g, 92\%); IR (thin film) v 3343, 3020, 2937, 1697, 1506, $1452 \mathrm{~cm}^{-1} ;{ }^{1} \mathrm{H} \mathrm{NMR}\left(400 \mathrm{MHz}, \mathrm{CDCl}_{3}\right) \delta 1.58(\mathrm{~s}$, $6 \mathrm{H}), 1.75-1.84(\mathrm{~m}, 1 \mathrm{H}), 2.04-2.09(\mathrm{~m}, 1 \mathrm{H}), 2.81-2.92(\mathrm{~m}, 4 \mathrm{H})$, $4.30(\mathrm{~d}, J=5.88 \mathrm{~Hz}, 2 \mathrm{H}), 5.00(\mathrm{brs}, 1 \mathrm{H}), 5.09(\mathrm{~s}, 1 \mathrm{H})$, 7.23-7.31 (m, 5H) ppm; $\left.{ }^{13} \mathrm{C} \mathrm{NMR} \mathrm{(100} \mathrm{MHz,} \mathrm{CDCl}_{3}\right) \delta 25.1$, 26.2, 31.2, 44.8, 57.6, 82.4, 127.5, 127.6, 128.7, 138.8, 155.3 ppm; HRMS (ESI) $m / z$ : $[\mathrm{M}+\mathrm{Na}]^{+}$calcd for $\mathrm{C}_{15} \mathrm{H}_{21} \mathrm{NO}_{2} \mathrm{~S}_{2} \mathrm{Na}$, 334.0911; found, 334.0905. 
2-(1,3-Dithian-2-yl)propan-2-yl butylcarbamate (5b): Pale yellow oil $(0.072 \mathrm{~g}, 89 \%)$; IR (thin film) $v 3359,2930,1710$, $1516 \mathrm{~cm}^{-1} ;{ }^{1} \mathrm{H}$ NMR $\left(400 \mathrm{MHz}, \mathrm{CDCl}_{3}\right) \delta 0.87(\mathrm{t}, J=7.24 \mathrm{~Hz}$, $3 \mathrm{H}), 1.27-1.36(\mathrm{~m}, 2 \mathrm{H}), 1.43-1.45(\mathrm{~m}, 2 \mathrm{H}), 1.54(\mathrm{~s}, 6 \mathrm{H})$, $1.75-1.82(\mathrm{~m}, 1 \mathrm{H}), 2.02-2.08(\mathrm{~m}, 1 \mathrm{H}), 2.79-2.92(\mathrm{~m}, 4 \mathrm{H})$, $3.06-3.11(\mathrm{~m}, 2 \mathrm{H}), 4.62$ (brs, $1 \mathrm{H}), 5.04(\mathrm{~s}, 1 \mathrm{H}) \mathrm{ppm} ;{ }^{13} \mathrm{C} \mathrm{NMR}$ $\left(100 \mathrm{MHz}, \mathrm{CDCl}_{3}\right) \delta 13.9,20.0,25.1,26.2,31.2,32.1,40.6$, 57.7, 81.9, $155.3 \mathrm{ppm}$; HRMS (ESI) $\mathrm{m} / z$ : $[\mathrm{M}+\mathrm{Na}]^{+}$calcd for $\mathrm{C}_{12} \mathrm{H}_{23} \mathrm{NO}_{2} \mathrm{~S}_{2} \mathrm{Na}$, 300.1068; found, 300.1056 .

2-(1,3-Dithian-2-yl)propan-2-yl allylcarbamate (5c): Orange solid (0.055 g, 72\%). mp 67-68 ${ }^{\circ} \mathrm{C}$; IR (thin film) $v 3343,3080$, 2940, 1707, $1516 \mathrm{~cm}^{-1} ;{ }^{1} \mathrm{H} \mathrm{NMR}\left(400 \mathrm{MHz}, \mathrm{CDCl}_{3}\right) \delta 1.54(\mathrm{~s}$, $6 \mathrm{H}), 1.70-1.81(\mathrm{~m}, 1 \mathrm{H}), 1.99-2.08(\mathrm{~m}, 1 \mathrm{H}), 2.79-2.91(\mathrm{~m}, 4 \mathrm{H})$, 3.70-3.72 (m, 2H), 4.76 (brs, 1H), 5.05-5.08 (m, 2H), 5.17 (d, $J=17.3 \mathrm{~Hz}, 1 \mathrm{H}), 5.74-5.84(\mathrm{~m}, 1 \mathrm{H}) \mathrm{ppm} ;{ }^{13} \mathrm{C} \mathrm{NMR}$ $\left(100 \mathrm{MHz}, \mathrm{CDCl}_{3}\right) \delta 25.1,26.2,31.2,43.2,57.5,82.2,116.0$, 134.7, $155.1 \mathrm{ppm}$; HRMS (ESI) $\mathrm{m} / z$ : $[\mathrm{M}+\mathrm{K}]^{+}$calcd for $\mathrm{C}_{11} \mathrm{H}_{19} \mathrm{NO}_{2} \mathrm{~S}_{2} \mathrm{~K}$, 300.0494; found, 300.0496 .

2-(1,3-Dithian-2-yl)propan-2-yl (2-((tert-butyldiphenylsilyl)oxy)ethyl)carbamate (5d): Colorless oil (0.142 g, 97\%); IR (thin film) $v$ 3369, 3076, 2930, 1713, 1510, $1452 \mathrm{~cm}^{-1}$; ${ }^{1} \mathrm{H}$ NMR (400 MHz, $\left.\mathrm{CDCl}_{3}\right) \delta 1.04(\mathrm{~s}, 9 \mathrm{H}), 1.57(\mathrm{~s}, 6 \mathrm{H})$, 1.74-1.83 (m, 1H), 2.04-2.11 (m, 1H), 2.82-2.92 (m, 4H), $3.27-3.31(\mathrm{~m}, 2 \mathrm{H}), 3.69-3.71(\mathrm{~m}, 2 \mathrm{H}), 4.96$ (brs, $1 \mathrm{H}), 5.07(\mathrm{~s}$, 1H), 7.34-7.41 (m, 6H), 7.62-7.64 (m, 4H) ppm; ${ }^{13} \mathrm{C}$ NMR $\left(100 \mathrm{MHz}, \mathrm{CDCl}_{3}\right) \delta 19.4,25.1,26.2,27.0,31.1,43.1,57.6$, 63.2, 82.2, 127.7, 129.9, 133.5, 135.7, 155.3 ppm; HRMS (ESI) $m / z:[\mathrm{M}+\mathrm{Na}]^{+}$calcd for $\mathrm{C}_{26} \mathrm{H}_{37} \mathrm{NO}_{3} \mathrm{~S}_{2} \mathrm{SiNa}, 526.1882$; found, 526.1875 .

General procedure for dM-Dmoc protection of arylamines synthesis of carbamates $5 \mathbf{e}-\mathbf{h}$ : To a solution of diisopropylamine (0.076 mL, $0.541 \mathrm{mmol}, 2.1$ equiv) in THF $(10 \mathrm{~mL})$ at $-78{ }^{\circ} \mathrm{C}$ under argon was added $n$-BuLi $(2.5 \mathrm{M}$ in pentane, $0.206 \mathrm{~mL}, 0.514 \mathrm{mmol}, 2$ equiv). The mixture was stirred for $15 \mathrm{~min}$. To the freshly prepared LDA solution was added an amine $\left(0.257 \mathrm{mmol}, 1\right.$ equiv) in THF $(50 \mathrm{~mL})$ at $-78^{\circ} \mathrm{C}$. After stirring for $45 \mathrm{~min}$, solid 4 ( $0.088 \mathrm{~g}, 0.257 \mathrm{mmol}, 1$ equiv) was added to the amide solution at $-78{ }^{\circ} \mathrm{C}$ under positive argon pressure. The mixture was stirred for $8 \mathrm{~h}$ while warming to $\mathrm{rt}$ gradually. The reaction was quenched with sat. $\mathrm{NH}_{4} \mathrm{Cl}(15 \mathrm{~mL})$ and extracted with EtOAc $(10 \mathrm{~mL} \times 2)$. The extracts were dried over anhydrous $\mathrm{Na}_{2} \mathrm{SO}_{4}$, filtered, and concentrated. The carbamates 5e (column eluted with 9:1 hexanes/EtOAc; TLC $R_{\mathrm{f}}=0.2$, developed with 5:1 hexanes/EtOAc), $\mathbf{5 f}(6: 1$ hexanes/ EtOAc; $R_{\mathrm{f}}=0.4$, 3:1 hexanes/EtOAc), 5g (5:1 hexanes/EtOAc; $R_{\mathrm{f}}=0.5,2: 1$ hexanes/EtOAc), and $\mathbf{5 h}(98: 2$ EtOAc/MeOH; $\left.R_{\mathrm{f}}=0.5,9: 1 \mathrm{EtOAc} / \mathrm{MeOH}\right)$ were purified with flash column chromatography $\left(\mathrm{SiO}_{2}\right)$.
2-(1,3-Dithian-2-yl)propan-2-yl phenylcarbamate (5e): Brown oil (0.035 g, 46\%); IR (thin film) v 3359, 3002, 2927, 1780, 1592, $1487 \mathrm{~cm}^{-1} ;{ }^{1} \mathrm{H} \mathrm{NMR}\left(400 \mathrm{MHz}, \mathrm{CDCl}_{3}\right) \delta 1.43(\mathrm{~s}$, $6 \mathrm{H}), 1.68-1.77(\mathrm{~m}, 1 \mathrm{H}), 2.02-2.08(\mathrm{~m}, 1 \mathrm{H}), 2.80-2.91(\mathrm{~m}, 4 \mathrm{H})$, $5.09(\mathrm{~s}, 1 \mathrm{H}), 7.21-7.33(\mathrm{~m}, 4 \mathrm{H}) \mathrm{ppm} ;{ }^{13} \mathrm{C} \mathrm{NMR}(100 \mathrm{MHz}$, $\left.\mathrm{CDCl}_{3}\right) \delta 24.6,26.1,31.1,56.3,85.3,127.8,128.2,128.6$, 138.7, $151.0 \mathrm{ppm}$; HRMS (ESI) $\mathrm{m} / \mathrm{z}:[\mathrm{M}+\mathrm{K}]^{+}$calcd for $\mathrm{C}_{14} \mathrm{H}_{19} \mathrm{NO}_{2} \mathrm{~S}_{2} \mathrm{~K}, 336.0754$; found, 336.0760.

2-(1,3-Dithian-2-yl)propan-2-yl (4-nitrophenyl)carbamate (5f): Brown oil (37 mg, 42\%); IR (thin film) $v$ 3305, 3080, 2971, 1732, 1620, $1595 \mathrm{~cm}^{-1} ;{ }^{1} \mathrm{H}$ NMR (400 MHz, $\left.\mathrm{CDCl}_{3}\right)$ $\delta 1.65(\mathrm{~s}, 6 \mathrm{H}), 1.78-1.85(\mathrm{~m}, 1 \mathrm{H}), 2.08-2.12(\mathrm{~m}, 1 \mathrm{H})$, 2.85-2.92 (m, 4H), $5.02(\mathrm{~s}, 1 \mathrm{H}), 6.95(\mathrm{brs}, 1 \mathrm{H}), 7.50(\mathrm{~d}$, $J=9.2 \mathrm{~Hz}, 2 \mathrm{H}), 8.17(\mathrm{~d}, J=9.2 \mathrm{~Hz}, 2 \mathrm{H}) \mathrm{ppm} ;{ }^{13} \mathrm{C} \mathrm{NMR}$ $\left(100 \mathrm{MHz}, \mathrm{CDCl}_{3}\right) \delta 25.1,26.2,31.3,57.4,84.6,117.8,125.3$, 143.0, 144.1, $151.2 \mathrm{ppm}$; HRMS (ESI) $\mathrm{m} / \mathrm{z}:[\mathrm{M}+\mathrm{Na}]^{+}$calcd for $\mathrm{C}_{14} \mathrm{H}_{18} \mathrm{~N}_{2} \mathrm{O}_{4} \mathrm{~S}_{2} \mathrm{Na}$, 365.0605; found, 365.0610 .

2-(1,3-Dithian-2-yl)propan-2-yl (pyridin-2-yl)carbamate (5g): Pale brown oil (0.043 g, 57\%); IR (thin film) $v 3184$, 2930, 1719, 1640, $1583 \mathrm{~cm}^{-1} ;{ }^{1} \mathrm{H}$ NMR (400 MHz, $\left.\mathrm{CDCl}_{3}\right)$ $\delta 1.63(\mathrm{~s}, 6 \mathrm{H}), 1.75-1.82(\mathrm{~m}, 1 \mathrm{H}), 2.02-2.08(\mathrm{~m}, 1 \mathrm{H})$, 2.80-2.88 (m, 4H), $5.14(\mathrm{~s}, 1 \mathrm{H}), 6.90-6.92(\mathrm{~m}, 1 \mathrm{H}), 7.61-7.65$ (m, 1H), $7.93(\mathrm{~d}, J=8.4 \mathrm{~Hz}, 1 \mathrm{H}), 8.33-8.35(\mathrm{~m}, 1 \mathrm{H}), 9.70$ (brs, 1H) $\mathrm{ppm} ;{ }^{13} \mathrm{C} \mathrm{NMR}\left(100 \mathrm{MHz}, \mathrm{CDCl}_{3}\right) \delta 25.3,26.2,31.3$, 57.2, 83.5, 112.8, 118.4, 138.4, 147.8, 152.3, 152.5 ppm; HRMS (ESI) $m / z:[\mathrm{M}+\mathrm{Na}]^{+}$calcd for $\mathrm{C}_{13} \mathrm{H}_{18} \mathrm{~N}_{2} \mathrm{O}_{2} \mathrm{~S}_{2} \mathrm{Na}$, 321.0707; found, 321.0701 .

2-(1,3-Dithian-2-yl)propan-2-yl (pyridin-4-yl)carbamate (5h): Orange oil (0.040 g, 52\%); IR (thin film) v 3170, 2950, 1719, 1636, $1580 \mathrm{~cm}^{-1} ;{ }^{1} \mathrm{H} \mathrm{NMR}\left(400 \mathrm{MHz}, \mathrm{CDCl}_{3}\right) \delta 1.63(\mathrm{~s}$, $6 \mathrm{H}), 1.76-1.84(\mathrm{~m}, 1 \mathrm{H}), 2.06-2.12(\mathrm{~m}, 1 \mathrm{H}), 2.84-2.91(\mathrm{~m}, 4 \mathrm{H})$, $5.01(\mathrm{~s}, 1 \mathrm{H}), 7.39(\mathrm{~d}, J=6.5 \mathrm{~Hz}, 2 \mathrm{H}), 7.55$ (brs, $1 \mathrm{H}), 8.39$ (d, $J=6.2 \mathrm{~Hz}, 2 \mathrm{H}) \mathrm{ppm} ;{ }^{13} \mathrm{C} \mathrm{NMR}\left(100 \mathrm{MHz}, \mathrm{CDCl}_{3}\right) \delta 24.9$, 26.1, 31.3, 57.3, 84.5, 112.9, 146.8, 149.2, 151.3 ppm; HRMS (ESI) $m / z:[\mathrm{M}+\mathrm{H}]^{+}$calcd for $\mathrm{C}_{13} \mathrm{H}_{18} \mathrm{~N}_{2} \mathrm{O}_{2} \mathrm{~S}_{2} \mathrm{H}, 299.0887$; found, 299.0879 .

$N$-((2-(1,3-Dithian-2-yl)propan-2-yl)oxy)carbonyl)-Lphenylalanine (5i): This compound was prepared following the general procedure for $\mathrm{dM}$-Dmoc protection of aliphatic amines with slight modifications: DMSO instead of THF was used as the solvent. During work-up, 5\% citric acid (instead of sat. $\mathrm{NH}_{4} \mathrm{Cl}$ ) and EtOAc were used for partition. Flash column chromatography $\left(\mathrm{SiO}_{2}, 3: 1: 0.04\right.$ hexanes/EtOAc/AcOH) gave 5i as a white foam after co-evaporation with toluene (80\%): TLC $R_{\mathrm{f}}=0.2(1: 1: 0.02$ hexanes $/ \mathrm{EtOAc} / \mathrm{AcOH}) ; \mathrm{IR}$ (thin film) $v$ 3331, 3035, 2933, 1715, $1497 \mathrm{~cm}^{-1} ;{ }^{1} \mathrm{H}$ NMR (400 MHz, $\left.\mathrm{CDCl}_{3}\right)$ two rotamers, $\delta 1.43(\mathrm{~s}, 1.2 \mathrm{H}), 1.45(\mathrm{~s}, 1.2 \mathrm{H}), 1.51(\mathrm{~s}$, 
$1.8 \mathrm{H}), 1.55(\mathrm{~s}, 1.8 \mathrm{H}), 1.74-1.79(\mathrm{~m}, 1 \mathrm{H}), 2.03-2.07(\mathrm{~m}, 1 \mathrm{H})$, 2.84-2.87 (m, 4H), 2.98-3.08 (m, 1H), 3.15-3.20 (m, 1H), $4.44-4.50(\mathrm{~m}, 0.4 \mathrm{H}), 4.57-4.62(\mathrm{~m}, 0.6 \mathrm{H}), 4.84(\mathrm{~s}, 0.4 \mathrm{H}), 5.02$ (s, 0.6H), 5.17-5.18 (m, 1H), $6.47(\mathrm{bs}, 1 \mathrm{H}), 7.18-7.28(\mathrm{~m}, 5 \mathrm{H})$ ppm; ${ }^{13} \mathrm{C} \mathrm{NMR}\left(100 \mathrm{MHz}, \mathrm{CDCl}_{3}\right)$ two rotamers, $\delta 24.8,25.1$, $26.2,31.2,38.1,39.3,54.6,57.5,83.2,84.3,127.2,128.7$, 129.7, 135.8, 154.5, 155.6, 175.9, $176.3 \mathrm{ppm}$; HRMS (ESI) $\mathrm{m} / \mathrm{z}$ : $[\mathrm{M}-\mathrm{H}]^{-}$calcd for $\mathrm{C}_{17} \mathrm{H}_{22} \mathrm{NO}_{4} \mathrm{~S}_{2}, 368.0996$; found, 368.0987 .

\section{General procedure for deprotection of dM-Dmoc protected} amines: To a suspension of 5 (1 equiv) in $\mathrm{THF} / \mathrm{H}_{2} \mathrm{O}(\mathrm{v} / \mathrm{v}$ 1:1) was added $\mathrm{NaIO}_{4}$ (10 equiv) at rt. After stirring overnight, the mixture was concentrated on a rotary evaporator. The residue was dissolved in acetone $(5 \% \mathrm{AcOH}$ in acetone for $\mathbf{5 i})$, and the insoluble inorganic salts were removed by filtration. The filtrate was concentrated on a rotary evaporator. The residue (after co-evaporation with toluene for $\mathbf{5 i}$ ) was suspended in methanol (dissolved in 1:1 methanol $/ \mathrm{H}_{2} \mathrm{O}$ in the case of 5i). Finely ground $\mathrm{K}_{2} \mathrm{CO}_{3}$ (10 equiv) was added, and the mixture was stirred at $\mathrm{rt}$ for $1 \mathrm{~h}$. Insoluble salts were removed by filtration. The filtrate was concentrated on a rotary evaporator and purified via flash column chromatography $\left(\mathrm{SiO}_{2}\right)$. All amine products 3a-i were known and were indentified with TLC and NMR. Chromatography and TLC information: 3a (column eluted with 8.5:1:0.5 EtOAc/MeOH/Et $3 \mathrm{~N}$; TLC $R_{\mathrm{f}}=0.4$, 9:1 EtOAc/MeOH), 3b $\left(8.5: 1: 0.5 \mathrm{EtOAc} / \mathrm{MeOH} / \mathrm{Et}_{3} \mathrm{~N} ; R_{\mathrm{f}}=0.2,9: 1\right.$ EtOAc/MeOH), 3c (8.5:1:0.5 EtOAc/MeOH/Et $3 \mathrm{~N} ; R_{\mathrm{f}}=0.2,9: 1$ EtOAc/MeOH), 3d (9:0.5:0.5 EtOAc/MeOH/Et ${ }_{3} \mathrm{~N} ; R_{\mathrm{f}}=0.5,9: 1$ EtOAc/MeOH), 3e (9:1 hexanes/EtOAc; $R_{\mathrm{f}}=0.2,5: 1$ hexanes/ EtOAc), $3 f$ (5:1 hexanes/EtOAc; $R_{\mathrm{f}}=0.3,3: 1$ hexanes/EtOAc), $3 g$ (5:1 hexanes/EtOAc; $R_{\mathrm{f}}=0.4,2: 1$ hexanes/EtOAc), $3 \mathbf{h}$ (9.5:0.5 EtOAc/MeOH; $\left.R_{\mathrm{f}}=0.5,9: 1 \mathrm{EtOAc} / \mathrm{MeOH}\right), 3 \mathbf{i}$ $\left(R_{\mathrm{f}}=0.5,3: 1: 1 n-\mathrm{BuOH} / \mathrm{AcOH} / \mathrm{H}_{2} \mathrm{O}\right)$. Isolated yields were obtained for 3a-h (see Table 1). Yield of $\mathbf{3 i}$ was determined to be $41 \%$ using RP-HPLC (column: C-18, $5 \mu \mathrm{m}, 100 \AA$, $250 \times 3.20 \mathrm{~mm}$; solvent A: $0.1 \mathrm{M}$ triethylammonium acetate, 5\% acetonitrile; solvent $\mathrm{B}: 90 \%$ acetonitrile; gradient: time, 0-60 min, solvent B, 0-45\%; flow rate: $1 \mathrm{~mL} / \mathrm{min}$; detection: UV $257 \mathrm{~nm}$ ) by comparing with authentic sample.

Selective deprotection of $\mathrm{dM}$-Dmoc protected amine in the presence of a Boc protected amine: Compound 6 was prepared following a reported procedure [22]. The general procedure for $\mathrm{dM}-\mathrm{Dmoc}$ protection of aliphatic amines (i.e., the procedure for the synthesis of $\mathbf{5 a - d}$ ) was used to covert $\mathbf{6}$ to 7 . Compound 7 was purified with flash column chromatography $\left(\mathrm{SiO}_{2}, 1: 1\right.$ hexanes/EtOAc): Colorless oil $(55 \%)$; TLC $R_{\mathrm{f}}=0.2$ (1:1 hexanes/EtOAc); IR (thin film) v 3356, 2933, 1707, 1690, $1510 \mathrm{~cm}^{-1}$; ${ }^{1} \mathrm{H}$ NMR $\left(400 \mathrm{MHz}, \mathrm{CDCl}_{3}\right) \delta 1.41(\mathrm{~s}, 9 \mathrm{H}), 1.54(\mathrm{~s}$, $6 \mathrm{H}), 1.74-1.81(\mathrm{~m}, 1 \mathrm{H}), 2.03-2.09(\mathrm{~m}, 1 \mathrm{H}), 2.83-2.88(\mathrm{~m}, 4 \mathrm{H})$, $3.28-3.32(\mathrm{~m}, 4 \mathrm{H}), 3.50-3.53(\mathrm{~m}, 4 \mathrm{H}), 3.57(\mathrm{~s}, 4 \mathrm{H}), 5.04(\mathrm{~s}$,
1H) ppm; $\left.{ }^{13} \mathrm{C} \mathrm{NMR} \mathrm{(100} \mathrm{MHz,} \mathrm{CDCl}_{3}\right) \delta 25.1,26.2,28.6$, 31.1, 40.7, 57.6, 70.4, 79.5, 82.2, 155.4, 156.2 ppm; HRMS (ESI) $m / z:[\mathrm{M}+\mathrm{Na}]^{+}$calcd for $\mathrm{C}_{19} \mathrm{H}_{36} \mathrm{~N}_{2} \mathrm{O}_{6} \mathrm{~S}_{2} \mathrm{Na}, 475.1912$; found, 475.1898. Selective removal of dM-Dmoc in the presence of Boc in 7 to give 6 was achieved following the general procedure for deprotection of $\mathrm{dM}$-Dmoc protected amines. The product was purified by flash column chromatography $\left(\mathrm{SiO}_{2}\right.$, 9.5:0.5 DCM/MeOH; TLC $\left.R_{\mathrm{f}}=0.4,9: 1 \mathrm{DCM} / \mathrm{MeOH}\right)$. Isolated yield of $80 \%$ was obtained.

\section{Selective deprotection of dM-Dmoc and Fmoc protected} amine: The dM-Dmoc and Fmoc protected diamine 9 was prepared from 1,2-bis(2-aminothoxy)ethane. Compound 8 was synthesized using the general procedure for $\mathrm{dM}$-Dmoc protection of aliphatic amines and purified with flash column chromatography $\left(\mathrm{SiO}_{2}, 9: 0.5: 0.5 \mathrm{DCM} / \mathrm{MeOH} / \mathrm{Et}_{3} \mathrm{~N}\right)$ : Light yellow oil (64\%); TLC $R_{\mathrm{f}}=0.3(9: 1 \mathrm{DCM} / \mathrm{MeOH})$; IR (thin film) $v 3359$, 2937, 1713, $1519 \mathrm{~cm}^{-1} ;{ }^{1} \mathrm{H} \mathrm{NMR}\left(400 \mathrm{MHz}, \mathrm{CDCl}_{3}\right) \delta 1.51(\mathrm{~s}$, $6 \mathrm{H}), 1.69-1.76(\mathrm{~m}, 1 \mathrm{H}), 2.00-2.06(\mathrm{~m}, 1 \mathrm{H}), 2.80-2.89(\mathrm{~m}, 4 \mathrm{H})$, 3.05 (bs, 2H), 3.25 (bs, 2H), 3.50 (t, J = 4.7 Hz, 4H), 3.56 (s, $4 \mathrm{H}) \mathrm{ppm}, 5.00(\mathrm{~s}, 1 \mathrm{H}), 5.44(\mathrm{bs}, 1 \mathrm{H}) ;{ }^{13} \mathrm{C} \mathrm{NMR}(100 \mathrm{MHz}$, $\left.\mathrm{CDCl}_{3}\right) \delta 25.2,26.3,31.2,40.7,41.5,57.7,70.2,70.3,70.4$, 82.1, $155.3 \mathrm{ppm}$; HRMS (ESI) $\mathrm{m} / z$ : $[\mathrm{M}+\mathrm{H}]^{+}$calcd for $\mathrm{C}_{14} \mathrm{H}_{28} \mathrm{~N}_{2} \mathrm{O}_{4} \mathrm{~S}_{2} \mathrm{H}, 353.1569$; found, 353.1576. For the synthesis of 9, compound $8(0.225 \mathrm{~g}, 0.640 \mathrm{mmol}, 1$ equiv) in 1,4dioxane $(5 \mathrm{~mL})$ and $15 \% \mathrm{Na}_{2} \mathrm{CO}_{3}(5 \mathrm{~mL})$ was reacted with Fmoc-Cl (0.402 g, 0.292 mmol, 1 equiv) at rt under argon. After $8 \mathrm{~h}$, the reaction mixture was diluted with EtOAc $(50 \mathrm{~mL})$, and the organic and aqueous phases were separated. The former was washed with water $(25 \mathrm{~mL}), 5 \%$ citric acid $(25 \mathrm{~mL})$ and brine $(25 \mathrm{~mL} \times 2)$, dried over anhydrous $\mathrm{Na}_{2} \mathrm{SO}_{4}$, filtered, and concentrated to dryness. The residue was purified with flash column chromatography $\left(\mathrm{SiO}_{2}, 1: 1\right.$ hexanes/EtOAc): Light yellow oil $(0.185 \mathrm{~g}, 50 \%)$; TLC $R_{\mathrm{f}}=0.2$ (1:1 hexanes/EtOAc); IR (thin film) $v 3337,3070,2943,1713,1449 \mathrm{~cm}^{-1}$; ${ }^{1} \mathrm{H}$ NMR $\left(400 \mathrm{MHz}, \mathrm{CDCl}_{3}\right) \delta 1.45(\mathrm{~s}, 6 \mathrm{H}), 1.69-1.79(\mathrm{~m}, 1 \mathrm{H})$, 2.00-2.04 (m, 1H), 2.77-2.85 (m, 1H), 3.26-3.29 (m, 2H), $3.36-3.37(\mathrm{~m}, 2 \mathrm{H}), 3.49-3.53(\mathrm{~m}, 4 \mathrm{H}), 3.56(\mathrm{~s}, 4 \mathrm{H}), 4.17-4.20$ $(\mathrm{m}, 1 \mathrm{H}), 4.37-4.38(\mathrm{~m}, 2 \mathrm{H}), 5.02(\mathrm{~m}, 1 \mathrm{H}), 5.16(\mathrm{bs}, 1 \mathrm{H}), 5.36$ (bs, 1H), 7.27 (t, J = 7.4 Hz, 2H), 7.35 (t, J = 7.4 Hz, 2H), 7.56 $(\mathrm{d}, \mathrm{J}=6.7 \mathrm{~Hz}, 2 \mathrm{H}), 7.72(\mathrm{~d}, \mathrm{~J}=7.4 \mathrm{~Hz}, 2 \mathrm{H}) \mathrm{ppm} ;{ }^{13} \mathrm{C} \mathrm{NMR}$ $\left(100 \mathrm{MHz}, \mathrm{CDCl}_{3}\right) \delta 25.2,26.3,31.2,40.7,41.2,47.5,57.7$, $68.8,70.3,70.5,82.2,120.1,125.2,127.1,127.8,141.4,144.1$, 155.3, $156.6 \mathrm{ppm}$; HRMS (ESI) $\mathrm{m} / z:[\mathrm{M}+\mathrm{H}]^{+}$calcd for $\mathrm{C}_{29} \mathrm{H}_{38} \mathrm{~N}_{2} \mathrm{O}_{6} \mathrm{~S}_{2} \mathrm{H}, 575.2250$; found, 575.2262. For selective removal of the Fmoc group of 9 to give 8,9 (90 $\mathrm{mg}$, $0.157 \mathrm{mmol}, 1$ equiv) in dry DCM $(10 \mathrm{~mL})$ was reacted with piperidine $(2 \mathrm{~mL})$ at $\mathrm{rt}$ under argon for $2 \mathrm{~h}$. The reaction mixture was concentrated to dryness under reduced pressure. The residue was purified with flash column chromatography $\left(\mathrm{SiO}_{2}\right.$, 9:0.5:0.5 DCM/MeOH/Et ${ }_{3} \mathrm{~N} ;$ TLC $\left.R_{\mathrm{f}}=0.3,9: 1 \mathrm{DCM} / \mathrm{MeOH}\right)$ 
to give 8 as a light yellow oil (45 mg, $82 \%$ ). For selective removal of the dM-Dmoc group of 9 to give $\mathbf{1 0}$ [23], the general procedure for deprotection of dM-Dmoc protected amines was used. The product was purified with flash column chromatography $\left(\mathrm{SiO}_{2}, 9: 0.5: 0.5 \mathrm{DCM} / \mathrm{MeOH} / \mathrm{Et}_{3} \mathrm{~N}\right.$, TLC $R_{\mathrm{f}}=0.5,8: 1: 1$ $\left.\mathrm{DCM} / \mathrm{MeOH} / \mathrm{Et}_{3} \mathrm{~N}\right)$. An isolated yield of $75 \%$ was obtained.

\section{Supporting Information}

\section{Supporting Information File 1}

Images of ${ }^{1} \mathrm{H}$ and ${ }^{13} \mathrm{C}$ NMR spectra of new compounds including 5a-i and 7-9.

[https://www.beilstein-journals.org/bjoc/content/ supplementary/1860-5397-14-149-S1.pdf]

\section{Acknowledgements}

Financial support from NIH (R15GM109288), NSF (CHE1111192), and PHF Graduate Assistantship (S.S.); the assistance from D. W. Seppala (electronics), J. L. Lutz (NMR), L. R. Mazzoleni (MS), M. Khaksari (MS), and A. Galerneau (MS); and NSF equipment grants (CHE1048655, CHE9512455, AGS1531454) are gratefully acknowledged.

\section{ORCID ${ }^{\circledR}$ iDs}

Shahien Shahsavari - https://orcid.org/0000-0001-7731-0785

Mark Sylvester - https://orcid.org/0000-0003-4472-7896

Emily Bromley - https://orcid.org/0000-0002-7160-7323

Savannah Joslin - https://orcid.org/0000-0002-2876-4032

Shiyue Fang - https://orcid.org/0000-0002-6523-7557

\section{References}

1. Wuts, P. G. M.; Greene, T. W. Protecting Groups in Organic Synthesis, 4th ed.; John Wiley \& Sons: Hoboken, New Jersey, 2007.

2. Ragnarsson, U.; Grehn, L. RSC Adv. 2013, 3, 18691-18697. doi:10.1039/c3ra42956c

3. Cavelier, F.; Enjalbal, C. Tetrahedron Lett. 1996, 37, 5131-5134. doi:10.1016/0040-4039(96)01070-2

4. Deng, J.; Hamada, Y.; Shioiri, T. J. Am. Chem. Soc. 1995, 117, 7824-7825. doi:10.1021/ja00134a035

5. Vuljanic, T.; Bergquist, K.-E.; Clausen, H.; Roy, S.; Kihlberg, J. Tetrahedron 1996, 52, 7983-8000. doi:10.1016/0040-4020(96)00369-9

6. Bu, X.; Xie, G.; Law, C. W.; Guo, Z. Tetrahedron Lett. 2002, 43, 2419-2422. doi:10.1016/S0040-4039(02)00237-X

7. Rao, T. S.; Nampalli, S.; Sekher, P.; Kumar, S. Tetrahedron Lett. 2002, 43, 7793-7795. doi:10.1016/S0040-4039(02)01759-8

8. Mazaleyrat, J.-P.; Xie, J.; Wakselman, M. Tetrahedron Lett. 1992, 33, 4301-4302. doi:10.1016/S0040-4039(00)74244-4

9. Hasan, A.; Stengele, K.-P.; Giegrich, H.; Cornwell, P.; Isham, K. R.; Sachleben, R. A.; Pfleiderer, W.; Foote, R. S. Tetrahedron 1997, 53, 4247-4264. doi:10.1016/S0040-4020(97)00154-3

10. Matsubara, N.; Oiwa, K.; Hohsaka, T.; Sadamoto, R.; Niikura, K.; Fukuhara, N.; Takimoto, A.; Kondo, H.; Nishimura, S.-I. Chem. - Eur. J. 2005, 11, 6974-6981. doi:10.1002/chem.200500531
11. Igarashi, J.; Kobayashi, Y. Tetrahedron Lett. 2005, 46, 6381-6384. doi:10.1016/j.tetlet.2005.06.171

12. Smith, A. B.; Safonov, I. G.; Corbett, R. M. J. Am. Chem. Soc. 2001, 123, 12426-12427. doi:10.1021/ja012220y

13. Barthels, R.; Kunz, H. Angew. Chem., Int. Ed. Engl. 1982, 21, 292. doi:10.1002/anie.198202921

14. Barthels, R.; Kunz, H. Angew. Chem. 1982, 94, 302. doi:10.1002/ange.19820940422

15. Kunz, H. Chem. Ber. 1976, 109, 3693-3706. doi:10.1002/cber.19761091123

16. Kunz, H.; Barthels, R. Chem. Ber. 1982, 115, 833-845. doi:10.1002/cber.19821150302

17. Waldmann, H.; Kunz, H. J. Org. Chem. 1988, 53, 4172-4175. doi:10.1021/jo00253a003

18. Lin, X.; Chen, J.; Shahsavari, S.; Green, N.; Goyal, D.; Fang, S. Org. Lett. 2016, 18, 3870-3873. doi:10.1021/acs.orglett.6b01878

19. Shahsavari, S.; Wigstrom, T.; Gooding, J.; McNamara, C.; Fang, S. Tetrahedron Lett. 2018, 59, 1763-1766. doi:10.1016/j.tetlet.2018.03.076

20. Shahsavari, S.; Gooding, J.; Wigstrom, T.; Fang, S. ChemistrySelect 2017, 2, 3959-3963. doi:10.1002/slct.201700364

21. Barnhurst, L. A.; Wan, Y.; Kutateladze, A. G. Org. Lett. 2000, 2, 799-801. doi:10.1021/ol005537w

22. Tang, W.; Fang, S. Tetrahedron Lett. 2008, 49, 6003-6006. doi:10.1016/j.tetlet.2008.07.174

23. Koda, H.; Brazier, J. A.; Onishi, I.; Sasaki, S. Bioorg. Med. Chem. 2015, 23, 4583-4590. doi:10.1016/j.bmc.2015.05.056

\section{License and Terms}

This is an Open Access article under the terms of the Creative Commons Attribution License (http://creativecommons.org/licenses/by/4.0). Please note that the reuse, redistribution and reproduction in particular requires that the authors and source are credited.

The license is subject to the Beilstein Journal of Organic Chemistry terms and conditions: (https://www.beilstein-journals.org/bjoc)

The definitive version of this article is the electronic one which can be found at: $\underline{\text { doi:10.3762/bjoc. } 14.149}$ 